\title{
COOPERACIÓN UNIVERSITARIA \\ PARA EL DESARROLlo EN MAKENI \\ (SiERra LeOna)
}

\section{Luis Perea Moreno}

Arquitecto y economista, profesor de la USPCEU Madrid. Coordinador de Cooperación al Desarrollo y del Plan Estratégico de Desarrollo Urbano en Makeni, Sierra Leona

\author{
Natalia García Fernández \\ Arquitecta, Máster en Arquitectura del Paisaje y experta \\ en Cooperación para el desarrollo
}

\section{Adela Salas Ruiz}

Arquitecta y experta en cooperación para el desarrollo

RESUMEN: Las ciudades medias africanas de menos de 500.000 habitantes representan el principal desafío urbano del momento a nivel mundial. Su desarrollo implicará, en las próximas décadas, un notable incremento de suelo urbano en los países en vías de desarrollo.

La falta de recursos, de un marco normativo adecuado, de profesionales en planificación urbana y los déficits de habitabilidad ya existentes en estos entornos, son realidades que evidencian, entre otras, la urgencia de articular un importante esfuerzo colectivo.

El planeamiento urbano y territorial convencional no es capaz de responder a estos retos en tiempo y escala. Por una parte, por la velocidad vertiginosa de la progresiva toma de tierra y el incesante crecimiento de la población y, por otra, debido a la dificultad que supone para la población asumir propuestas de planificación con visos de futuro en contextos en los que no existe cultura en planeamiento.

Así, entre Madrid (España) y Makeni (Sierra Leona) surge una experiencia en la que la Universidad y la Cooperación al Desarrollo emergen como ele- 
mentos críticos de fortalecimiento institucional y formativo. La colaboración entre universidades (USPCEU-UNIMAK) es el germen de un proceso de reflexión urbana que implica decididamente al Ayuntamiento y a los principales actores locales de la ciudad.

Palabras clave: desarrollo humano sostenible, expansión urbana, planeamiento estratégico, ciudades africanas, procesos participativos.

RESUM: Les ciutats mitjanes africanes de menys de 500.000 habitants representen el principal desafiament urbà del moment a nivell mundial. El seu desenvolupament implicarà, en les pròximes dècades, un notable increment de sòl urbà en els països en vies de desenvolupament.

La manca de recursos, d'un marc normatiu adequat, de professionals en planificació urbana i els dèficits d'habitabilitat ja existents en aquests entorns, són realitats que evidencien, entre altres, la urgència d'articular un important esforç col·lectiu.

El planejament urbà i territorial convencional no és capaç de respondre a aquests reptes en temps i escala. D'una banda, per la velocitat vertiginosa de la progressiva presa de terra i l'incessant creixement de la població i, de l'altra, a causa de la dificultat que suposa per a la població assumir propostes de planificació amb aspectes de futur en contextos en què no hi cultura en planejament.

Així, entre Madrid (Espanya) i Makeni (Sierra Leone) sorgeix una experiència en la qual la Universitat i la Cooperació al Desenvolupament emergeixen com a elements crítics d'enfortiment institucional i formatiu. La col-laboració entre universitats (USPECEU-UNIMAK) és el germen d'un procés de reflexió urbana que implica decididament l'Ajuntament i als principals actors locals de la ciutat.

Paraules ClaU: desenvolupament humà sostenible, expansió urbana, planejament estratègic, ciutats africanes, processos participatius.

ABSTRACT: Medium-sized african cities, with fewer than 500,000 inhabitants are the main urban challenge of the moment. In the coming decades, 
urban development will involve a significant increase in urban land in developing countries.

The lack of resources, an appropriate regulatory framework, professionals in urban planning and habitability in urban environments of these cities, are realities that show the urgency for to articulate a major collective effort.

Conventional urban and regional planning is not able to respond to these challenges in time and scale. On the one hand because of the speed of the progressive increase of illegal settlements and the constant population growth. On the other hand on account of the difficulty for people to take proposals forward planning in contexts where there is no culture in planning.

Between Madrid (Spain) and Makeni (Sierra Leone) starts an experience in which the University and Development Cooperation emerge as critical elements of institutional and educational strengthening and training. Collaboration between universities (USPCEU-UNIMAK) is the beginning of a process of urban reflection involving the City Council and local stakeholders.

KEYWORDS: Sustainable human development, urban development, strategic plan, african cities, participatory processes.

\section{La colaboración entre universidades para la cooperación al desarrollo. Relación entre universidades con miradas comunes hacia Makeni}

L as universidades europeas se enfrentan al reto de formar y capacitar a los futuros profesionales de un mundo cada vez más globalizado e introducir en sus planes de estudios los temas relacionados con el Desarrollo Humano Sostenible como una oferta atractiva y demandada por los alumnos.

Con la vocación de introducir la cooperación al desarrollo en el programa universitario, surge la iniciativa de formalizar relaciones académicas entre universidades de distintas latitudes, no como un añadido sino como un 
pilar básico a impulsar dentro de las enseñanzas técnicas. Desde este punto de partida esencial, el proyecto que se presenta nace del entorno universitario y se traslada a la realidad de la mano de un proceso largo y cauteloso que avanza implicando nuevos actores.

Esta investigación es una suma de muchos y diferentes esfuerzos que, bajo el título Apoyo al Fortalecimiento de la Universidad de Makeni, Sierra Leona, inicia su andadura en 2009 con una colaboración entre la Universidad CEU San Pablo de Madrid (USPCEU-Madrid), la Universidad de Makeni (UNIMAK) y el Ayuntamiento de Makeni, con el apoyo de la Escuela Técnica Superior de Arquitectura de Madrid (ETSAM) y el Instituto de Cooperación en Habitabilidad Básica - Cátedra UNESCO de Habitabilidad Básica en la UPM - Madrid, (ICHAB) como un proceso que ha ido extendiéndose desde la universidad a otros ámbitos de la ciudad de Makeni; una gran alianza entre universidades, ciudadanía de Makeni y administración central.

Esta iniciativa docente se ha conformado, ya desde sus inicios, como una IAP, un proyecto de Investigación-Acción Participativa a escala universitaria y a escala de ciudad, con el fin de generar conocimientos y capacidades en todos los implicados de distintas ramas técnicas y sociales. Está diseñada con el propósito de construir una red de alumnado, profesorado e investigadores españoles y sierraleoneses que orienten su profesión a los aspectos vinculados con la habitabilidad en las urbes africanas, y en concreto en el África Occidental, con Makeni, Sierra Leona, como modelo de referencia y pruebas. Un método de investigación y aprendizaje colectivo, que parte de análisis críticos desde el norte en los que se implican de manera activa los grupos desde el sur, actuando, no sólo como meros objetos de investigación, sino como parte primordial del proceso, la base que contribuye al conocimiento y germen de transformación de su propia realidad.

Esta relación pretende poder ser una herramienta de transformación abierta, a través de la participación e implicación de todos los agentes, que, con la ciudad informal africana como escenario de fondo, permita otorgar capacidades a los futuros profesionales para responder de manera eficiente en contextos desfavorecidos. 


\section{De la investigación a la consolidación de un grupo de trabajo multidisciplinar}

La experiencia docente se ha engarzado en diferentes asignaturas y actividades de la formación académica universitaria. Desde asignaturas troncales, optativas, workshops, charlas... se ha fomentado el trabajo y la reflexión vinculada a la ciudad de Makeni, en una dinámica que se nutre de forma importante con los distintos viajes realizados a Sierra Leona. La experiencia docente se sitúa en el marco de un proyecto más amplio, un proyecto en el que con el Desarrollo Humano Sostenible como bandera se pretende contribuir a la lucha contra la pobreza y la promoción del desarrollo social, político y económico del norte de Sierra Leona y de la ciudad de Makeni en concreto. Todo ello a través del fortalecimiento de la Universidad de Makeni unido al apoyo del Ayuntamiento de Makeni, y mediante la implicación de nuevas instituciones y actores del territorio fomentando el trabajo y aprendizaje conjunto.

Se trata de un proyecto transversal que engloba distintas áreas del conocimiento dentro de la arquitectura y el urbanismo, y extensible también a las ingenierías, en un afán por crear un grupo multidisciplinar técnico que dé un apoyo global a la ciudad. Debido a la íntima relación entre UNIMAK y el Ayuntamiento de Makeni, se incluyó entre las propuestas la redacción de un Plan Estratégico para la ciudad de Makeni.

Esta colaboración, en el campo de la arquitectura y el urbanismo, se apoya en dos ejes transversales prioritarios; la ciudad informal africana y la exploración de nuevas formas de abordar desde el planeamiento territorial los retos de la expansión acelerada, y los nuevos modelos de construcción e infraestructuras aplicables a dichos contextos.

Formar profesionales capaces de responder con una visión global del mundo a las necesidades de la ciudadanía, es un compromiso que la universidad debe asumir. En contextos africanos en los que, al igual que Sierra Leona, no existe la formación académica de áreas afines a la arquitectura y el urbanismo, los problemas derivados de la ocupación del suelo se vuelven extremos siendo necesario el apoyo a la universidad, reforzando e implementando sus capacidades educativas con el fin de que puedan tener sus propios profesionales para afrontar su futuro. Por lo tanto, esta irrefutable relación 
reafirma la formación como elemento clave para el desarrollo convirtiendo la universidad en un espacio de aplicación directa en la realidad.

\section{De las relaciones universitarias al fortalecimiento institucional}

El proyecto se concibe desde el fortalecimiento de las capacidades educativas de la Universidad de Makeni (Sierra Leona) que implica la relación innegable de igual a igual entre universidades. La Universidad de Makeni (UNIMAK), siendo referencia institucional en la ciudad, se convierte en la llave de cualquier acción actuando como contraparte desde el sur.

El esfuerzo realizado por UNIMAK para reunir en el proyecto a los principales actores locales supone un impulso muy importante y refuerza su papel en la sociedad y el gobierno de la ciudad. UNIMAK fomenta la implicación de su alumnado en los distintos proyectos e insta a los ciudadanos a que sean partícipes del proyecto. Esta relación lleva a una visión a largo plazo, y al convencimiento de que la incidencia efectiva pasa por un proceso de acompañamiento trabajando con el acervo de la población.

La relación con las instituciones locales, en este caso con el Ayuntamiento, conlleva una aproximación directa a la realidad por lo que es importante que el compromiso de la universidad africana sea fuerte, actuando como articulación entre los actores locales. Además, fortalece la confianza de la ciudadanía en las instituciones locales, propiciando su participación en la vida social, política y económica de la ciudad.

\section{Del fortalecimiento institucional al planeamiento urbano y territorial en Makeni}

El proceso, articulado desde un importante compromiso participativo, ha permitido a la población entender y rubricar, en los distintos foros realizados en la ciudad de Makeni, la necesidad de marcar unas líneas estratégicas de ordenación territorial, que permitan controlar y pautar los futuros crecimientos de su ciudad, ayudando a anticipar los principales retos futuros. 
En este contexto surge el Plan Estratégico de Desarrollo Urbano de Makeni, una propuesta de planificación, un proceso de suma de miradas con un horizonte común, de consensos, en avance..., una mirada al futuro desde la reflexión sobre la gestión óptima de sus valores y sensible a los recursos económicos colectivos e individuales. La estrategia implica una decidida aproximación integral, que pone en valor la capacidad productiva del territorio, permitiendo compatibilizar la protección de las áreas naturales con un desarrollo urbano y socioeconómico sostenible. Desde la detección de prioridades inmediatas (infraestructuras básicas, salud, educación, zonas vulnerables...) el plan avanza un marco de futuro flexible para el debate apoyándose en el valor de su territorio y de su paisaje.

\section{Hacia una planificación territorial y urbana para Makeni. Retos y dificultades}

Sierra Leona (África Occidental), con un 39,50\% de población urbana, es un país rico en recursos minerales y pesqueros, si bien muy poco desarrollado y con una distribución de la riqueza muy desigual. Casi dos tercios de la población depende de una agricultura de subsistencia, que se nos presenta como un exponente fundamental para el desarrollo del país.

Makeni, la quinta ciudad del país, con una población de 115.000 habitantes, es la capital de la provincia de Bombali, la más pobre de Sierra Leona. Una urbe africana de tamaño medio con lógicas urbanas, periurbanas y rurales que ha experimentado unos crecimientos de población exponenciales pudiendo llegar a duplicar la población en el año 2035.

Considerando estimaciones recientes, con una tasa del 3,2\% de crecimiento, Makeni albergaría 25.000 nuevos habitantes en 2020 y unos 45.000 más si el crecimiento es del 5,6\%. Estos datos implican un consumo de suelo muy importante a considerar.

Un contexto de gran crecimiento urbano que incrementará las notables carencias existentes en la ciudad, en cuanto a acceso a la vivienda, infraestructuras básicas, sanidad, formación técnica..., que demanda con urgencia articular unas trazas básicas que den respuesta a estas necesidades. 
Este desarrollo urbano actual sigue patrones dispersos sin planificación alguna en el que los procesos de crecimiento se apoyan en normas tradicionales. El suelo es propiedad de un pequeño número de familias, herencia de un pasado lejano, en el que la figura del Paramount Chief realiza la tarea de custodia de las tierras y sin la aprobación del cual no es posible realizar ningún tipo de actuación que les afecte. El territorio en torno a la ciudad central se configura por ciudades satélites en las que los problemas, vinculados a la habitabilidad, se acrecientan.

La principal dificultad del planeamiento en Makeni responde a los altos riesgos derivados de la ocupación actual del territorio, que obliga a anticiparse a los retos de la expansión acelerada desde procesos estratégicos participativos.

El desarrollo urbano de Makeni se cristaliza casa a casa en áreas de muy baja densidad cuyo incremento progresivo evidencia graves problemas y riesgos a futuro. Principalmente los derivados de la ocupación de áreas vulnerables (inundaciones, riadas...); el crecimiento sin provisión de empleo, infraestructuras y equipamientos; el impacto en el Medioambiente y alteración de ecosistemas (deforestación, erosión...); la segregación social (ciudad formal/ciudad informal); las dificultades en la articulación a futuro del entorno metropolitano (fusión de ciudades); los futuros costes en el mejoramiento (2,6 veces más costoso el mejoramiento que la urbanización de terrenos)... que deben ser tenidos en cuenta no sólo como puntos de partida, sino por lo que suponen para la población estas dinámicas tan arraigadas a su idiosincrasia.

Los vertiginosos tiempos de la expansión de las ciudades africanas no entienden de planificación. Los instrumentos occidentales convencionales no son válidos aquí.

Frente a una realidad compleja, es esencial reconocer y asumir el proceso histórico de transformación del lugar y prever una capacidad de carga realista fundamentada en criterios de interés general, racional y sostenible, de forma que pueda ser partícipe la población.

También es fundamental abordar las prioridades más inmediatas, definir acciones realistas y jerarquizadas en un marco integral coherente, que permita también anticipar en lo posible los desafíos que los veloces ritmos de 
crecimiento urbano imponen. Poder anticipar respuestas apoyadas en nuevas figuras y metodologías adaptadas, es crítico, como se reconoce repetidamente desde diferentes esferas técnicas a nivel internacional.

El diseño de una herramienta de ámbito territorial, que pueda ser asumida como propia por la población, debe amoldarse a una realidad específica con lógicas propias de la sociedad, donde la carencia de técnicos especializados y la falta de experiencia global en planeamiento deben hacer especialmente sensible todo el proceso. En este sentido, es esencial integrar la capacitación a los agentes de decisión e implicados en la toma de decisiones como un componente más de la estrategia.

\section{El Plan Estratégico de Desarrollo Urbano de Makeni}

En este contexto de precariedad habitacional, de un marco normativo insuficiente, de una realidad social vinculada a costumbres muy arraigadas ajenas a una planificación de futuro y de insuficiencia de recursos técnicos y económicos, se inician los primeros contactos entre el USPCEU, UNIMAK y el Ayuntamiento de Makeni, donde se hace explícita la necesidad de abrir vías de reflexión conjunta en relación a la ciudad de Makeni y su entorno a futuro, el inicio de un proceso de planeamiento estratégico participativo.

En julio 2013 y enero 2014 se realizan 2 grandes foros en la ciudad de Makeni, con todas las partes implicadas presentes (universidades, administración y grupos locales de acción), que ponen sobre la mesa las aproximaciones a los principales problemas urbanos de Makeni.
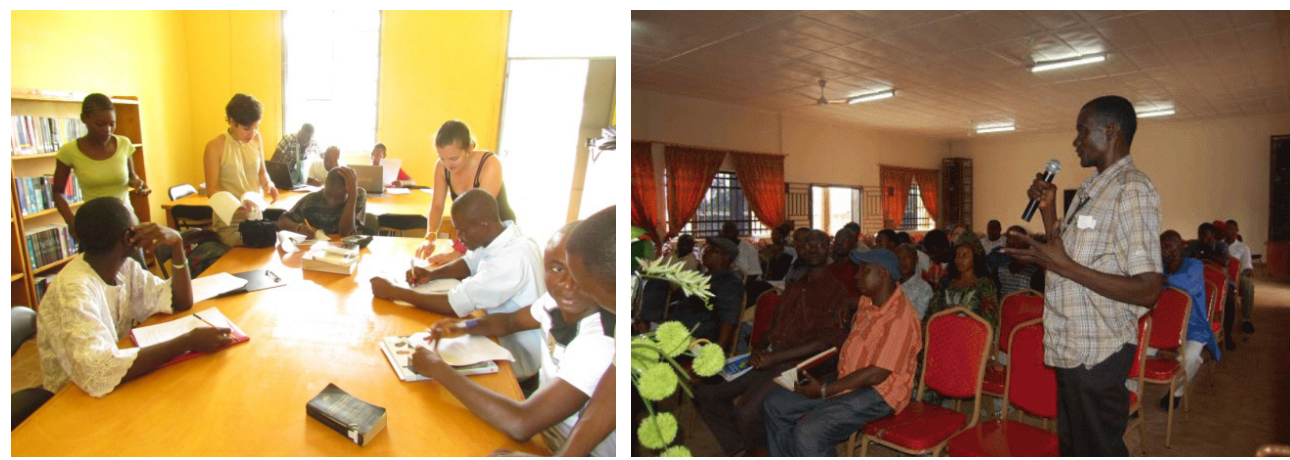

Imágenes 1 y 2. Foros participativos de Makeni. Hacia un Makeni sostenible, julio de 2013 


\section{Hacia un Makeni Sostenible. Primer Foro de Participación Ciudadana}

En julio de 2013, en el primer foro participativo Towards a Sustainable Makeni, se asientan las bases para el proceso de planeamiento estratégico participativo y se sintetizan las principales reflexiones sobre la ciudad.

Este primer encuentro, desarrollado en las dependencias municipales, supone un gran esfuerzo colectivo en los 2 días de duración. Sobre una estructura de trabajo en 5 áreas temáticas (Gobernanza y Participación; Medio Ambiente y Biodiversidad; Infraestructuras y Equipamientos; Planeamiento Urbano, Movilidad y Calidad de Vida; y Actividades Económicas, Desarrollo y Cohesión Social), los participantes se organizaron en grupos mixtos (con representación de universidades de Madrid y Makeni, personal del ayuntamiento, del distrito, colectivos sociales, Paramount Chief...), que expusieron sobre cada tema elementos críticos y líneas de acción futuras.

La principal conclusión del I Foro de Participación Ciudadana es la evidencia de definir para la ciudad un modelo de plan urbano, un modelo de ciudad que englobe respuestas desde el urbanismo (compacidad y definición de centros urbanos, densificación y definición de usos...) con encuadres territoriales (modelos de ciudad central y ciudades satélites, optimización de los usos del suelo, definición de los crecimientos urbanos, actividad económica generadora, definición de infraestructuras básicas y supramunicipales y preservación del medio) a los principales problemas de Makeni.

Los pasos a seguir, consensuados tras el primer foro, serían: la creación de un comité de planeamiento urbano capaz de responder a las futuras proyecciones de población en 20 o 30 años, el desarrollo de un diagnóstico de la situación actual de Makeni, el inicio de un catastro, y desarrollar las primeras ideas a futuro buscando un Makeni sostenible y el interés por un segundo foro, en continuación de éste. 

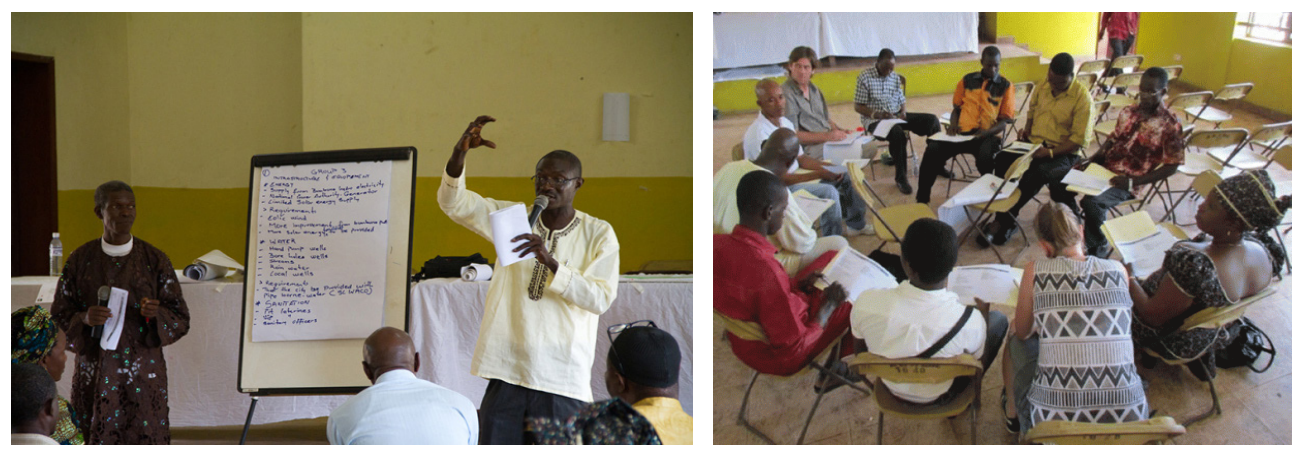

Imágenes 3 y 4. Foros participativos de Makeni. Hacia un Makeni sostenible, julio de 2013

\section{Hacia un Plan Estratégico para Makeni. Segundo Foro de Participación Ciudadana}

En el segundo foro participativo, en enero de 2014, Towards a Strategic Urban Plan for Makeni, se presentan las primeras propuestas a debatir y se firma el acuerdo para la realización de un Plan Estratégico de Desarrollo Urbano para Makeni entre USPCEU-Madrid, UNIMAK y Ayuntamiento de Makeni.

Cabe destacar en este foro, la firma del acuerdo entre las 3 instituciones sobre un documento que supone en sí mismo la base esencial del proceso. Este documento, contiene información resumida de la normativa relativa al planeamiento, de los actores fundamentales, de los procesos de desarrollo urbano reciente, de la importancia de la planificación, una propuesta de fases para el proceso y unas primeras ideas globales a debatir.

La población ha entendido y rubricado en los 2 grandes foros, la necesidad de marcar unas líneas estratégicas de ordenación territorial a largo plazo, que, en espera de la creciente población que habrá de albergar, permitan controlar y pautar los futuros crecimientos de su ciudad.

Se evidencian, del mismo modo, las dificultades para la implementación de las propuestas en un contexto en el que la tierra pertenece a unas pocas familias, lo que implica una aparente traba al proceso. En cambio, la asistencia de jefes locales con intervenciones muy sensibles a la urgencia de la pla- 
nificación y la decidida apuesta de la administración local permiten albergar esperanzas en el futuro del proceso.

Las principales propuestas para el debate inicial en el entorno de Makeni y las 7 millas (espacio de acción que el Gobierno de Sierra Leona otorga a las ciudades medias como Makeni para controlar el territorio inmediato) fueron sintetizadas gráficamente en un deliberado esfuerzo esquemático para su mejor comprensión por todas las partes. En un territorio muy ajeno a la información cartográfica, la reflexión global fue considerada muy positiva y altamente valorada por los asistentes a las sesiones de trabajo.

\section{Reflexiones para el proceso de planificación}

Junto a las propuestas específicas que se presentaron para el debate, el documento que sirvió de firma, aporta unas decisiones de planeamiento adicional que se consideran claves, para la continuación del proceso:

- La coordinación entre todos los actores como elemento esencial de planificación estratégica.

- La división del territorio de Makeni en sectores operativos para su estudio, donde la zona central sería un área de intervención prioritaria

- La integración de las propuestas globales en el marco de las acciones y planes sectoriales y de todo tipo, en las esferas locales, regionales y nacionales.

- Delimitación de áreas restringidas no aptas para la urbanización, principalmente, en torno a los espacios de gran pendiente y las swamps o zonas inundables de la ciudad y en su territorio.

- Definición de áreas a preservar como zonas naturales o espacios libres en los que las swamps comienzan a vislumbrarse como espacios de oportunidad a futuro. En un contexto urbano donde lo público está casi ceñido a los viales, las swamps, saneadas y drenadas, se advierten como una potencial red de espacios libres a futuro, conectora de otros ámbitos naturales y públicos a mayor y menor escala.

- La reserva de áreas para equipamientos e infraestructuras territoriales. 
Las reflexiones finales sobre los encuentros participativos fueron muy positivas, evidenciándose la necesidad de apoyo técnico especializado en planeamiento urbano y demandando y reafirmando la necesidad de la implicación técnica desarrollada desde Madrid, lo que supone un refuerzo del proceso realizado y de este modelo de Cooperación Universitaria al Desarrollo, con implicación en Planificación Urbanística y Territorial desde las instituciones.

\section{De los foros participativos a la formalización de las principales estrategias para el territorio de Makeni}

El proceso de trabajo colectivo entre instituciones tras la firma del acuerdo en Makeni sigue su recorrido. El trabajo de reflexión urbana supone un salto importante, tanto en la definición de las propuestas y avances, como en la producción de nuevos materiales analíticos de enorme valor para la comprensión del territorio.

El trabajo desarrollado desde Madrid se sintetiza y remite a UNIMAK y al Ayuntamiento de Makeni con el fin de poder evaluarlos junto con los principales implicados en unos talleres participativos de enfoque técnico en Makeni. Estos talleres de conocimiento del territorio y de evaluación de propuestas técnicas se han visto pospuestos por la emergencia sanitaria que ha supuesto la llegada del ébola a Sierra Leona, pero se mantiene el compromiso de ser retomados cuando se pueda volver.

\section{Principales estrategias para el territorio de Makeni}

Desde una aproximación especialmente sensible a los valores del territorio y a la estructura de su paisaje, se comienza un minucioso trabajo de planeamiento territorial, apoyado en los principios esenciales del planeamiento estratégico (agilidad, adaptación al contexto, participación, jerarquía de acciones, flexibilidad...) y en la consideraciones obtenidas del trabajo conjunto con el Ayuntamiento de Makeni y los principales actores, profundizando y ajustando las acciones y propuestas incluyendo nuevas prioridades. Los principales avances en el trabajo se apoyan en el concepto de Infraestructura Verde como base para la reflexión y genera un avance importante en el proceso. 
Algunas de las principales estrategias sobre las que se trabaja en la actualidad son:

- La definición de la Infraestructura Verde de Makeni. La identificación en el territorio de una red de espacios libres de edificación que engloben las áreas vulnerables, los espacios de mayor capacidad productiva, los espacios identitarios..., trabajando en su planificación y adecuación a uso público para definir donde albergar áreas protegidas y áreas oportunas para el incremento de la agricultura, la reforestación o la urbanización.

- La integración de los futuros polos equipados e infraestructuras en el territorio. Las infraestructuras producen impactos territoriales por lo que es fundamental analizar su integración en el medio para evitar una excesiva fragmentación del territorio. Por ello, pautar y ordenar los espacios alrededor de las infraestructuras con la mínima vulnerabilidad, dando prioridad a los corredores ya consolidados y la compatibilidad armónica entre éstas y el territorio, resulta clave.

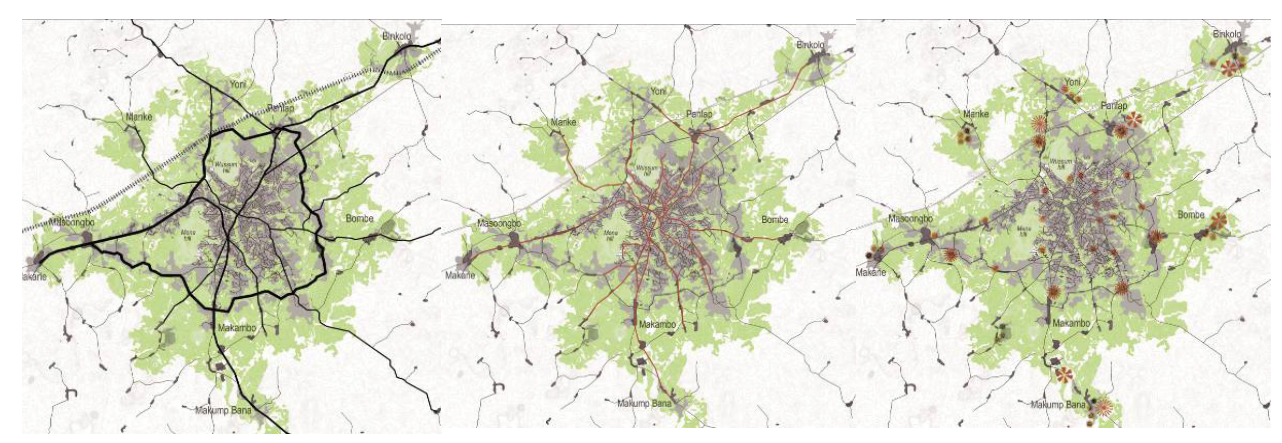

Imágenes 5, 6 y 7. Infraestructura Verde y posibles áreas de reserva para el crecimiento de la ciudad: propuestas de red de comunicaciones, red de infraestructuras básicas y red de equipamientos

- Mejorar la competitividad de actividad agrícola. La priorización de actividades agrícolas que garanticen el aprovechamiento de los recursos naturales de Makeni como vía para un esperado desarrollo económico, con la preservación de los suelos con mayor capacidad productiva y el aprovechamiento máximo de las posibilidades de terciarización 
agrícola debe ser un pilar prioritario. Así, trabajar en el desarrollo de programas de cooperativas de gestión que permitan priorizar el mantenimiento de la actividad agropecuaria con el fin de impulsar actuaciones vinculadas a explotación colectiva, a través de asociaciones de productores y de cooperativas agrarias facilitando el desarrollo productivo de las mismas.

- Pautar los futuros crecimientos urbanos del territorio de Makeni para los próximos 35 años. La definición de un programa de gestión, a futuro, ha de permitir identificar acciones, la planificación por fases y cuantificación en el espacio y en el tiempo. Es necesaria la identificación de escenarios y una propuesta flexible por fases, para el desarrollo de acciones y crecimiento de la ciudad.

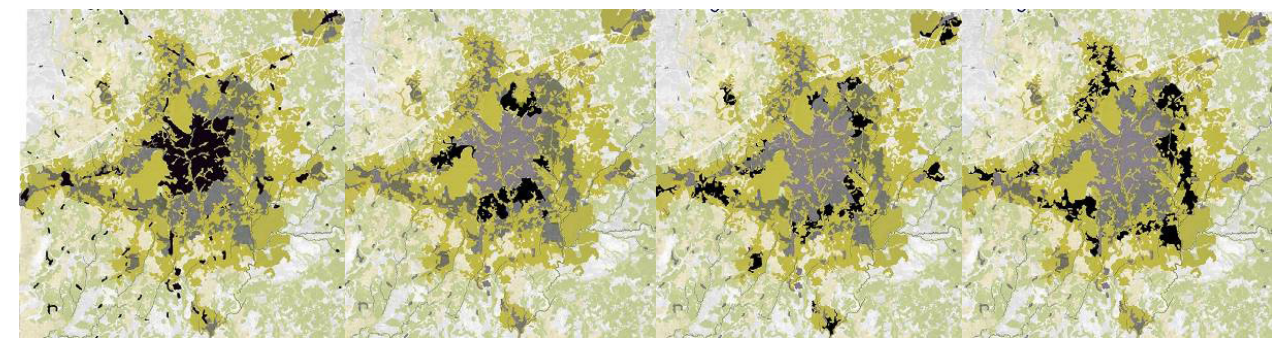

Imagen 8. Ocupación del suelo, crecimientos urbanos y jerarquización de acciones: áreas de regeneración, consolidación, de extensión y reserva urbana

\section{Conclusiones y consideraciones finales}

\section{La extensión de la cooperación entre universidades a otras áreas e instituciones: los agentes locales como herramienta de gestión territorial}

La cooperación entre universidades, como vínculo con el resto de agentes locales es la clave en este proceso. La Universidad se convierte en un agente fundamental para la Cooperación al Desarrollo con la finalidad de implementar las capacitaciones y generar conciencia crítica en todas las partes implicadas, desde una base totalmente técnica y rigurosa. Desde las asig- 
naturas, grupos de trabajo e investigación, profesorado y alumnado... tienen el enorme potencial de apoyo continuo desde los conocimientos específicos aportando donde éstos escasean.

\section{La formación como elemento transversal crítico}

Dentro de los procesos de colaboración institucional que, como éste, suponen un apoyo técnico en territorios que lo demandan, la articulación de estrategias formativas paralelas a los profesionales locales resulta clave. Desde la Universidad como soporte para la implementación de líneas académicas futuras en planeamiento urbano, poder dar el paso al desarrollo de acciones formativas directamente vinculadas a los trabajos realizados y que se están llevando a cabo son algunas líneas de inicio. En este sentido, el Plan Estratégico, concebido como un camino conjunto abierto, integra esta componente en la voluntad de que, en un futuro cercano, la cooperación universitaria se convierta en colaboración.

\section{El conocimiento de la realidad como base}

La interacción con las esferas locales debe ser constante. En cada viaje al territorio de Makeni, nuevos elementos, realidades, datos, claves... van apareciendo. Es esencial realizar un recorrido minucioso y atento de todo lo que sucede. Los valores tradicionales (grupos y asociaciones tradicionales, lugares sagrados, ritmos...), la estructura de la tierra, sus dinámicas y actores, las estructuras jerárquicas de gobierno social y su importancia como actores reconocidos en el desarrollo, las dificultades normativas y técnicas, la necesidad de implementar esfuerzos gráficos adaptados son algunos de los temas fundamentales que solo desde un trabajo de largo recorrido y desde el lugar, es posible comprender.

\section{Los tempos del planeamiento participativo: la importancia del proceso y la visión de largo plazo}

La identificación de valores y fortalezas del territorio por parte de su población debe partir de un reconocimiento conjunto de éste y de un conocimiento mutuo de todas las partes implicadas, con visión a largo plazo. 
Los procesos en los que la implicación ciudadana es clave son sin duda más lentos y costosos que otros. Esto es un proceso no definido de antemano, en el que no puede primar el cumplimiento de los objetivos, sino la detonación de procesos asumiendo las estructuras sociales, esbozando un planeamiento participativo en el que no hay una sola forma de hacer cosas.

\section{Por un planeamiento estratégico versus un planeamiento legislativo}

La ciudad planificada como producto acabado de lógicas occidentales es inconcebible en un contexto en el que la principal problemática es la accesibilidad a los recursos. El plan urbanístico que sólo legisla y delimita suelos se torna aquí utópico y tirano por igual. Sólo cabe la evolución de un plano urbano a un plan de estrategias. La evolución del Plan Municipal a un Plan Estratégico con visiones más generales y globales, que jerarquiza acciones y proyectos prioritarios que pueden ser implementados, se flexibiliza, identifica propuestas clave, es comprensible, participado, ágil en cuanto a los plazos y a la tramitación, es realista..., y no por ello deja de responder con rigor a todos los requerimientos iniciales.

\section{La replicabilidad del modelo}

Este modelo de cooperación en pro del desarrollo humano sostenible en otras latitudes es completamente replicable en cualquier escuela de Arquitectura o Ingeniería de la Unión Europea para trabajar en pro del fortalecimiento de una universidad africana actuando ésta de contraparte. Por una parte, como inicio de una nueva experiencia, por otra, como suma a un proceso ya iniciado. Un modelo en el que Universidades de Europa y de África trabajen juntas con el objetivo último de ofrecer una formación con una visión global a los futuros profesionales del mundo. 


\section{Referencias}

Busquets, J. (1999): La Urbanización Marginal, Universitat Politècnica de Catalunya, Barcelona.

Caminos, H. et al. (1984): Elementos de Urbanización, Gustavo Gili, México.

DAvis, M. (2014): Planeta de ciudades miseria, Akal, Madrid.

Martín, M; V. Díaz y E. Rodríguez (2014): Un modo de a frontar la ciudad africana, Catarata, Madrid.

Nogué, J; L. Puigbert; P. Sala y G. Bretcha (2010): Paisatge i participació ciutadana, Observatorio del Paisaje de Cataluña, Olot.

Rist, G. (2002): El desarrollo: historia de una creencia occidental, Catarata, Madrid.

SAChs, J. (2005): El Fin de la Pobreza, Debate, Madrid.

VvaA y M. Kabunda (coord.) (2011): África y la cooperación con el Sur desde el Sur, Catarata, Madrid.

Vvaa y Salas, J. (coord.) (2009): Habitáfrica. Cuatro realizaciones de habitabilidad básica en África, Fundación CEAR, Madrid.

VvaA (2008): «África», Dossier La vanguardia, La vanguardia, Barcelona.

VvaA (2009): «Arquitectos Sin recursos», Arquitectos, 186, CSCAE, Madrid.

VvaA (2009): Informe global sobre asentamientos humanos 2009, UN-Habitat, Earthscan, London.

VvaA (2013): Informe sobre Desarrollo Humano 2013, PNud, Programa de las Naciones Unidas para el Desarrollo, Nueva York.

VvaA (2010): Strategic Urban Development Plan for Masaka Municipality, UN-Habitat, Nairobi.

VvaA (2014): The State of African Cities, UN-Habitat, Nairobi.

VvaA (2010): Urban Manual Planning for Somaliland, UN-Habitat, Nairobi.

VvaA (2012): Urban Planning for city Leaders, UN-Habitat, Nairobi. 